\title{
Searching for massless Dark Photons at the LHC via Higgs production
}

\section{S. Biswas}

KIAS, Seoul, Republic of Korea

E-mail: sanjoy@kias.re.kr

\section{E. Gabrielli}

Università di Trieste, and INFN, Sezione di Trieste, Trieste, Italy, and NICPB, Tallinn, Estonia

E-mail: emidio.gabrielli@cern.ch

\section{Heikinheimo}

Helsinki Institute of Physics, University of Helsinki, Helsinki, Finland

E-mail: matti.heikinheimo@helsinki.fi

\section{B. Mele*}

INFN, Sezione di Roma, Rome, Italy

E-mail: barbara.mele@romal.infn.it

\begin{abstract}
Massless dark photons are predicted in hidden-sector models with an unbroken dark U(1) gauge symmetry. A particular class of these models, aiming to solve both the Yukawa-hierarchy and the dark-matter problems of the standard model, manifests natural Higgs nondecoupling properties for the dark photon. As a consequence, we show that the Higgs-boson production at colliders followed by the Higgs decay into a photon and a dark photon provides a very promising dark-photon production mechanism. This decay gives rise to an unconventional Higgs signature characterized by a resonating gamma-plus-missing-momentum system with a monochromatic photon. We discuss the sensitivity of the LHC to the corresponding signal for a Higgs boson produced in both gluon-fusion and vector-boson-fusion channels.
\end{abstract}

EPS-HEP 2017, European Physical Society conference on High Energy Physics 5-12 July 2017

Venice, Italy

\footnotetext{
*Speaker.
} 


\section{Introduction}

Most theoretical models introduced to solve the hierarchy problem of the Higgs scalar field in the standard model (SM) of particle interactions would require the observation of some hints of inconsistency and/or new physical states in collisions at the TeV energy scale. Despite that, in the first few years of running at 7, 8 and $13 \mathrm{TeV}$, the CERN Large Hadron Collider (LHC) has explored collisions at the $\mathrm{TeV}$ scale in many many different channels, and found up to now a global picture of consistency with the SM. While the predictions of the most popular beyond-the-standardmodel (BSM) models are being thoroughly scrutinized, and are presently sort of late in showing up, one should try to have a coverage as extensive as possible of new non-conventional experimental signatures in order to make the search for either new particles or inconsistencies (or both) as model independent as possible.

The class of models containing hidden (or dark) sectors (where hidden/dark means made up of fields uncharged under the SM gauge group) can indeed provide new signatures not yet covered by present searches. When including a dark $\mathrm{U}(1)$ gauge group, the dark sector predicts the existence of a dark photon. The way the dark photon can show up in experiments depends on its mass (in case the extra $\mathrm{U}(1)$ is broken), coupling and hidden-sector structure. Most attention in collider physics has been given up to now to the case of massive dark photons (see e.g. [1]), where the U(1) gauge field naturally develops a tree-level interaction with ordinary matter mediated by kineticmixing. In case the dark photon is massless the latter interaction can be rotated away [2, 3]. The dark-photon interaction with ordinary matter occurs then via higher-order operators, and is suppressed by typically large new-physics energy scales. Correspondingly, the search for massless dark photons must proceed via different phenomenological strategies with respect to the massive case.

We consider a particular class of hidden sectors, described in [4], that aims to explain the hierarchy in the Yukawa couplings of fermions in the SM, and, at the same time, can provide realistic dark-matter candidates. In this class of models, a nonperturbative mechanism involving a dark unbroken $\mathrm{U}(1)$ gauge symmetry generates an exponential hierarchy in the dark-fermion mass spectrum, that is in turn transmitted to the ordinary-fermion mass spectrum via a messenger sector. Dark fermions are only charged under the dark U(1) [with an integer-charge sequence], and might be natural dark-matter candidates. While the predicted massless dark photon $\bar{\gamma}$ has in general suppressed interactions with the ordinary matter, for typical values of the charges and mixing parameters of the model the Higgs boson can enter in a nondecoupling regime. Then, the one-loop interaction with the dark photon (mediated by the messengers) is not suppressed by a large energy scale but by just the Higgs-boson field vacuum expectation value $v[5,6]$. This regime gives rise to new peculiar signatures in the Higgs decays that could be observed in Higgs production at high-energy collisions. In the following, we will consider the corresponding phenomenology at the LHC.

\section{Higgs decay into massless dark photons}

The new Higgs boson decay channels involving a massless dark photon are $H \rightarrow \gamma \bar{\gamma}, Z \bar{\gamma}, \bar{\gamma} \bar{\gamma}$ [3]. In a nondecoupling regime, they can have sizeble rates [5]. The signature of a massless dark photon 


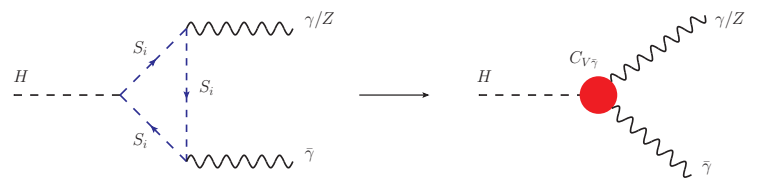

Figure 1: Effective $H \gamma \bar{\gamma}(H Z \bar{\gamma})$ interaction described as the low energy approximation of a one-loop diagram mediated by heavy messengers $S_{i}[6]$.

in collider experiments will be the same as the neutrino one, thanks to the suppressed interaction with ordinary matter, and to its massless nature. The Higgs decay into two dark photons will then affect the Higgs invisible decay width. Here we consider the single dark photon Higgs signature, as detailed in Figure 1, where the effective $H \gamma \bar{\gamma}(H Z \bar{\gamma})$ interaction is described as the low energy approximation of a one-loop diagram mediated by heavy messengers $S_{i}$. We focus in particular on the $H \rightarrow \gamma \bar{\gamma}$ channel giving rise to a resonating gamma-plus-missing-momentum signature, characterized by one monochromatic photon with energy $m_{H} / 2$, and same amount of missing energy. Note that the photon energy is more than twice the one in the resonance corresponding to the rare SM decay $H \rightarrow \gamma Z \rightarrow \gamma v \bar{v}$. While a quantitative prediction for the $H \rightarrow \gamma \bar{\gamma}$ decay width will be in general dependent on quite a few model parameters, in a simplified-model analysis [5] one can obtain typical allowed ranges which correspond to branching ratios (BRs) up to about 5\%. This is shown in Figure 2 [5], where $\mathrm{BR}(H \rightarrow \gamma \bar{\gamma})$ is plotted versus the dark $\mathrm{U}(1)$ fine structure constant $\bar{\alpha}$ (details on the model assumed and on the definition of relevant input parameters can be found in [5]). Such large $\mathrm{BR}(H \rightarrow \gamma \bar{\gamma})$ might indeed lead to an observable signal in Higgs production at the LHC, as discussed in the next section.

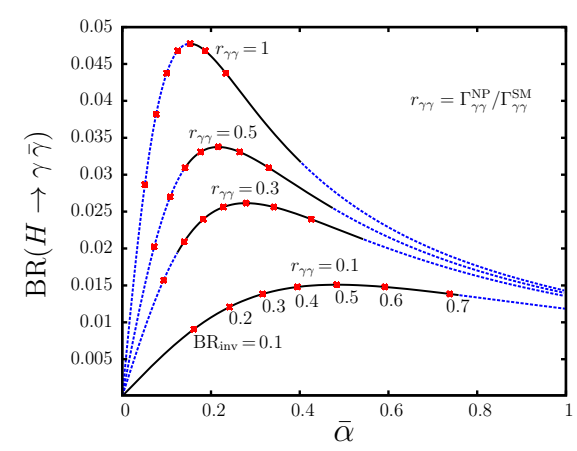

Figure 2: $\mathrm{BR}(H \rightarrow \gamma \bar{\gamma})$ versus the dark $\mathrm{U}(1)$ fine structure constant $\bar{\alpha}$. The black solid lines show the allowed BR ranges as a function of the relative new physics contribution to the $H \rightarrow \gamma \gamma$ width, and of the $H \rightarrow \bar{\gamma} \bar{\gamma}$ contribution to the Higgs invisible BR [5].

\section{Dark-photon production via Higgs production at the LHC}

In [5] the potential of the Run-1 dataset at the LHC has been studied at parton level by considering the gluon fusion process $g g \rightarrow H \rightarrow \gamma \bar{\gamma}$ at the c.m. proton energy of $8 \mathrm{TeV}$ with $20 \mathrm{fb}^{-1}$ of 
integrated luminosity. It is particularly informative to look at a comparison of the distribution of the missing transverse mass $M_{T}=\sqrt{2 p_{T}^{\gamma} E_{T}^{\text {miss }}(1-\cos \Delta \phi)}$ of the resonating $\gamma \bar{\gamma}$ system in the Higgs signal (where $\Delta \phi$ is the azimuthal distance between the photon and dark-photon three momenta) and of the main irreducible and reducible backgrounds, as shown in Figure 3 [5]. In that figure, the

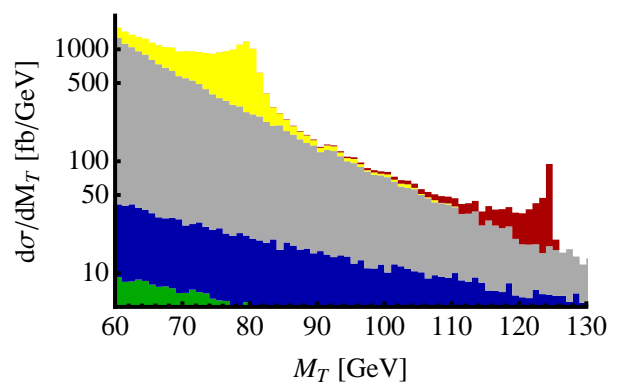

Figure 3: Distribution of the $\gamma \bar{\gamma}$ transverse invariant mass assuming $\mathrm{BR}(H \rightarrow \gamma \bar{\gamma}) \simeq 0.5 \%$ (red) compared to the main irreducible and reducible backgrounds, as from [5].

$\gamma+E_{T}^{\text {miss }}$ transverse invariant mass distribution (in $\mathrm{fb} / \mathrm{GeV}$ ) of the signal for $B R(H \rightarrow \gamma \bar{\gamma})=5 \%$ (shown in red) is compared with: $i$ ) the $\gamma j$ channel (grey) [where large apparent $E_{T}^{\text {miss }}$ is created by a combination of real $E_{T}^{\text {miss }}$ from neutrinos in heavy quark decays and mismeasured jet energy]; $i i$ ) the $\gamma Z \rightarrow \gamma v \bar{v}$ channel (blue); iii) $j Z \rightarrow j v \bar{v}$ (green), where the jet is misidentified as a photon; and iiii) $W \rightarrow e v$ (yellow), where the electron (positron) is misidentified as a photon. The resonant structure of the signal emerges clearly from the background. The definition of the relevant basic objects, corresponding cut-flows, and detection efficiencies applied in Figure 3 are described in [5]. With a parton-level event-generator analysis, one finds that a $5 \sigma$ discovery can be reached with the existing $8 \mathrm{TeV}$ LHC data-set down to $\mathrm{BR}(H \rightarrow \gamma \bar{\gamma}) \simeq 0.5 \%$ [5].

An improved analysis (including parton shower effects, and a more realistic background simulation) essentially confirms this estimate for the Run-1 potential [7]. When increasing the LHC collision energy up to $\sqrt{s}=14 \mathrm{TeV}$, beyond the gluon fusion channel also the vector-boson-fusion Higgs production channel deserves to be considered. A thorough analysis of the two channels with a comparison with the corresponding backgrounds at $14 \mathrm{TeV}$ has been carried out in [7]. In Table 1 , one can find the LHC sensitivity to $\mathrm{BR}(H \rightarrow \gamma \bar{\gamma})$ for different integrated luminosities in the two channels. One can see that the gluon-fusion process at $14 \mathrm{TeV}$ considerably extends the Run-1 potential, and turns out to be about one order of magnitude more sensitive than the vector-boson-fusion channel. In particular, for an integrated luminosity of 100 (300) $\mathrm{fb}^{-1}$ at $14 \mathrm{TeV}$, the signal significance $S / \sqrt{S+B}$ for the gluon-fusion channel approaches the $5 \sigma$ level at $\mathrm{BR}_{\gamma \bar{\gamma}}=\mathrm{BR}(H \rightarrow \gamma \bar{\gamma}) \simeq 1.6(0.9) \times 10^{-3}$. At the High-Luminosity LHC, with an integrated luminosity of $3 \mathrm{ab}^{-1}$, the $5 \sigma$ reach is extended down to $\mathrm{BR}_{\gamma \bar{\gamma}} \simeq 2.9 \times 10^{-4}$. On the other hand, in absence of any signal observation, a 95\% C.L. exclusion bound could be set at $\mathrm{BR}_{\gamma \bar{\gamma}}>(6.4,3.7,1.2) \times 10^{-4}$, with $(100,300,3000) \mathrm{fb}^{-1}$ of integrated luminosity, respectively.

Of course detailed detector-level studies will be needed to draw realistic conclusions on the LHC sensitivity to the new striking signature of a single monochromatic photon resonating with missing momentum that is associated to the Higgs decay into massless dark photons. 


\begin{tabular}{c|cc|cc|cc}
$\mathrm{BR}_{\gamma \bar{\gamma}}(\%)$ & \multicolumn{2}{|c|}{$\mathrm{L}=100 \mathrm{fb}^{-1}$} & \multicolumn{2}{|c|}{$\mathrm{L}=300 \mathrm{fb}^{-1}$} & \multicolumn{2}{|c}{$\mathrm{L}=3 \mathrm{ab}^{-1}$} \\
\hline Significance & $2 \sigma$ & $5 \sigma$ & $2 \sigma$ & $5 \sigma$ & $2 \sigma$ & $5 \sigma$ \\
\hline $\mathrm{BR}_{\gamma \bar{\gamma}}(\mathrm{VBF})$ & 0.76 & 1.9 & 0.43 & 1.1 & 0.14 & 0.34 \\
\hline $\mathrm{BR}_{\gamma \bar{\gamma}}(g g F)$ & 0.064 & 0.16 & 0.037 & 0.092 & 0.012 & 0.029 \\
\hline
\end{tabular}

Table 1: Reach in $\operatorname{BR}(H \rightarrow \gamma \bar{\gamma})$ (in percentage) for a 95\% C.L. exclusion and a $5 \sigma$ discovery at the 14 $\mathrm{TeV}$ LHC, for a Higgs produced either in the VBF or in the gluon-fusion channels, for different integrated luminosities L.

Finally, it should be stressed that, apart from LHC, other collider setups, like $e^{+} e^{-}$colliders or fixed-target experiments, can also be invaluable to look for footprints of a massless dark photon in high-energy collisions [6],[8]-[10].

Acknowledgments : The work of M.H. has been supported by the Academy of Finland project number 267842.

\section{References}

[1] G. Aad et al. [ATLAS Collaboration], A search for prompt lepton-jets in pp collisions at $\sqrt{s}=8 \mathrm{TeV}$ with the ATLAS detector, JHEP 1602 (2016) 062 [arXiv:1511.05542 [hep-ex]].

[2] B. Holdom, Two U(1)'s and Epsilon Charge Shifts, Phys. Lett. 166B (1986) 196.

[3] B. A. Dobrescu, Massless gauge bosons other than the photon, Phys. Rev. Lett. 94 (2005) 151802 [hep-ph/0411004].

[4] E. Gabrielli and M. Raidal, Exponentially spread dynamical Yukawa couplings from nonperturbative chiral symmetry breaking in the dark sector, Phys. Rev. D 89 (2014) no.1, 015008 [arXiv:1310.1090 [hep-ph]].

[5] E. Gabrielli, M. Heikinheimo, B. Mele and M. Raidal, Dark photons and resonant monophoton signatures in Higgs boson decays at the LHC, Phys. Rev. D 90 (2014) no.5, 055032 [arXiv:1405.5196 [hep-ph]].

[6] S. Biswas, E. Gabrielli, M. Heikinheimo and B. Mele, Dark-photon searches via ZH production at $e^{+} e^{-}$colliders, Phys. Rev. D 96 (2017) no.5, 055012 [arXiv:1703.00402 [hep-ph]].

[7] S. Biswas, E. Gabrielli, M. Heikinheimo and B. Mele, "Dark-Photon searches via Higgs-boson production at the LHC, Phys. Rev. D 93 (2016) no.9, 093011 [arXiv:1603.01377 [hep-ph]].

[8] S. Biswas, E. Gabrielli, M. Heikinheimo and B. Mele, Higgs-boson production in association with a dark photon in $e^{+} e^{-}$collisions, JHEP 1506 (2015) 102 [arXiv:1503.05836 [hep-ph]].

[9] E. Gabrielli, B. Mele, M. Raidal and E. Venturini, FCNC decays of standard model fermions into a dark photon, Phys. Rev. D 94 (2016) no.11, 115013 [arXiv:1607.05928 [hep-ph]].

[10] M. Fabbrichesi, E. Gabrielli and B. Mele, Hunting down massless dark photons in kaon physics, Phys. Rev. Lett. 119 (2017) no.3, 031801 [arXiv:1705.03470 [hep-ph]]. 